\title{
Comparison of body fatness measurements by near-infrared reactance and dual-energy $X$-ray absorptiometry in normal-weight and obese black and white women
}

\author{
Courtney L. Jennings ${ }^{1}$, Lisa K. Micklesfield ${ }^{1,2}$, Mike I. Lambert ${ }^{1}$, Estelle V. Lambert ${ }^{1}$, Malcolm Collins ${ }^{1,3}$ \\ and Julia H. Goedecke ${ }^{1,3 *}$ \\ ${ }^{1}$ UCT/MRC Research Unit for Exercise Science and Sports Medicine, Department of Human Biology, University of Cape Town, \\ PO Box 115, Newlands 7725, Cape Town, South Africa \\ ${ }^{2}$ MRC Mineral Metabolism Research Unit, Department of Paediatrics, University of Witwatersrand, Johannesburg, South Africa \\ ${ }^{3}$ South African Medical Research Council, Cape Town, South Africa
}

(Received 4 June 2009 - Revised 18 September 2009 - Accepted 21 September 2009 - First published online 2 November 2009)

The aim of the present study was to compare body fat percent (BF\%) using single-site near-IR reactance (NIR) and dual-energy X-ray absorptiometry (DXA) in a cohort of normal-weight $\left(\mathrm{BMI}<25 \mathrm{~kg} / \mathrm{m}^{2}\right)$ black $(n 102)$ and white $(n 71)$; and obese $\left(\mathrm{BMI} \geq 30 \mathrm{~kg} / \mathrm{m}^{2}\right)$ black ( $n$ 117) and white ( $n$ 41) South African women (18-45 years). NIR-derived BF \% was significantly correlated with DXA-derived BF \% in all groups: normal-weight black $(r 0.55,95 \%$ CI: $0.40,0.67, P<0.001)$ and white $(r 0.69,95 \%$ CI: $0.53,0.79, P<0 \cdot 001)$ women; obese black $(r 0.59,95 \% \mathrm{CI}: 0.46,0.70, P<0.001)$ and white $(r 0.56,95 \% \mathrm{CI}: 0.30,0.74, P<0.001)$ women. NIR under-predicted BF\% compared to DXA in black women (normal-weight, -4.36 (SD 4.13) \% and obese, -3.41 (SD 3.72)\%), while smaller mean differences were observed in white women (normal-weight, -0.29 (SD 4.19) \% and obese, -0.81 (SD 3.09)\%), irrespective of normal-weight or obese status $(P<0.001)$. In obese subjects, NIR-derived BF $\%$ did not measure values greater than approximately $45 \%$, while the maximum DXA-derived measure was $58 \%$. In conclusion, although there was a significant relationship between NIR- and DXA-derived BF\%, NIR under-predicted BF\% in normal-weight and obese black South African women compared to DXA, but to a greater extent in subjects with very high levels of adiposity $(>45 \%)$. The results of single-site NIR as a measure of BF\% should therefore be interpreted with caution, particularly in women of African descent and in those with very high levels of adiposity.

Ethnicity: Dual-energy X-ray absorptiometry: Body composition: Obesity

Obesity has become a global epidemic in both developed and developing countries ${ }^{(1)}$. In South Africa, urbanised women, in particular, are at an increased risk of developing obesity with more than $58 \%$ of black and $49 \%$ of white women classified as overweight or obese, based on $\mathrm{BMI}^{(2)}$. Since increased adiposity is associated with increased CVD risk $^{(3)}$, a rapid and cost effective method of quantifying body fat percent (BF\%) is becoming an increasingly important aspect of health risk appraisal and research.

Traditionally, BMI $\left(\mathrm{kg} / \mathrm{m}^{2}\right)$ has been used as a proxy measure of adiposity in health screening and in large epidemiological studies. However, the relationship between $\mathrm{BMI}$ and $\mathrm{BF} \%$ is influenced by factors such as muscularity and ethnicity, and therefore varies by population ${ }^{(4-7)}$. There are more precise methods of quantifying BF\%, such as dual-energy X-ray absorptiometry (DXA) ${ }^{(8)}$ and underwater weighing ${ }^{(9)}$. However, these methods are not always feasible for large-scale health screening and research due to the high cost and limited access to facilities, particularly in developing countries. In contrast, near-IR reactance (NIR) is a practical field measure of $\mathrm{BF} \%$, which may be used in situations where methods such as DXA and underwater weighing are not practical.

The validity and limits of agreement of NIR as a measure of $\mathrm{BF} \%$ have been shown to be population specific. A recent study comparing NIR to underwater weighing, has reported that NIR tended to over-estimate $\mathrm{BF} \%$ in lean individuals and under-estimate $\mathrm{BF} \%$ in individuals with higher levels of $\operatorname{adiposity}^{(10)}$. Studies in individuals with darker skin tone, including American Indian women ${ }^{(11)}$ and African American men ${ }^{(12)}$, found that NIR under-reported BF \% when compared to a criterion measure, such as underwater weighing. However, to our knowledge, there are no studies that have examined the ability of NIR to estimate BF\% in black African women. Studies in the USA and South Africa have shown that black African women have less visceral fat and greater peripheral fat compared to their white counterparts ${ }^{(13-15)}$, which may affect the validity of the measurement.

Abbreviations: BF \%, body fat percent; DXA, dual-energy X-ray absorptiometry; NIR, near-IR reactance.

* Corresponding author: Dr Julia H. Goedecke, fax +27 21686 7530, email Julia.goedecke@uct.ac.za 
Therefore, the aim of the present study was to compare BF \% measured using single-site NIR and DXA in a cohort of normal-weight and obese, black and white South African women.

\section{Methods}

\section{Subjects}

The study sample consisted of normal-weight (BMI $<25$ $\left.\mathrm{kg} / \mathrm{m}^{2}\right)$ black ( $n$ 102) and white $(n$ 71); and obese $\left(\mathrm{BMI} \geq 30 \mathrm{~kg} / \mathrm{m}^{2}\right)$ black $(n$ 117) and white $(n$ 41) South African women aged 18-45 years old, recruited from church groups, community centres and universities. Subjects were excluded from the study if they were pregnant or lactating, or had any known disease or were taking any medication that might affect body composition. The present study was conducted according to the guidelines laid down in the Declaration of Helsinki and all procedures were approved by the Research Ethics Committee of the Faculty of Health Sciences at the University of Cape Town. Written informed consent was obtained from all the subjects before participation in the study.

\section{Body composition assessment}

Weight (in light weight clothing without shoes) and height were measured. BF\% was measured by single-site NIR (Futrex-6100 A/ZL, Futrex Inc., Gaithersburg, MD, USA) as described previously ${ }^{(16)}$. Briefly, the subjects' sex, weight, height and age were entered into the NIR device that was then zero-adjusted according to the manufacturer's instructions. Each subject sat with her dominant arm relaxed on an examination table, while the light wand of the NIR was placed on the biceps muscle at the mid point between the elbow and the shoulder. The reading is determined via an IR light which penetrates approximately $1 \mathrm{~cm}$ into the tissue of the measurement site. Scans are made over a range of wavelengths from $700-1100 \mathrm{~nm}$ and the average of six optical density readings are used to derive BF \%. A NIR light shield was used to block out any surrounding light which could affect the measurement. The $\mathrm{CV}$ for $\mathrm{BF} \%$ measured by NIR on forty-five obese individuals on two occasions on the same day in our laboratory was $13.6 \%$ (SEE 2.6).

Whole body composition was assessed using DXA (Hologic QDR 4500 Discovery-W dual-energy X-ray absorptiometer, software version 4.40, Hologic Inc., Bedford, MA, USA) according to standard procedures, with an in vivo precision (percentage of $\mathrm{CV}$ ) of $1.7 \%$ for fat mass. The arm replacement method was used to measure whole body composition in those subjects that exceeded the scanning region ${ }^{(17)}$.

\section{Statistical analysis}

The data were analysed using the STATISTICA version 7 (StatsSoft Inc., Tulsa, OK, USA). All data are expressed as means and standard deviations. Differences in groups were compared using ANOVA, adjusting for age where appropriate. Pearson product-moment and Spearman's rank order correlations were used to describe the relationships between the two techniques. As there were no differences in the interpretation of the results, only Pearson correlation coefficients are reported. Limits of agreement between the two measurements of $\mathrm{BF} \%$ were determined using the technique of Bland \& Altman ${ }^{(18)}$. One-way ANOVA was used to determine if the bias in NIR- and DXA-derived BF \% was different by group. Significance was accepted at $P<0.05$.

Estimated sample size was calculated from the data of normal-weight white women assuming a minimum difference of $3 \%$ body fat between techniques and a standard deviation of $4 \%$ within a group (www.newstats.org). This calculation predicted a minimum sample size of twenty-nine subjects per group using a power of $80 \%(1-\beta)$ and an $\alpha$ level of $0 \cdot 05$. However, as the variation within the black obese and normal-weight subgroups was not known, this sample size was increased to guard against a potential lack of statistical power.

\section{Results}

\section{Subject characteristics}

The subject characteristics of the normal-weight and obese, black and white subjects are described in Table 1. The obese

Table 1. Basic characteristics of the black and white, normal-weight and obese South African women (Mean values and standard deviations)

\begin{tabular}{|c|c|c|c|c|c|c|c|c|}
\hline & \multicolumn{4}{|c|}{ Normal-weight $\left(\mathrm{BMI} \leq 25 \mathrm{~kg} / \mathrm{m}^{2}\right)$} & \multicolumn{4}{|c|}{ Obese $\left(\mathrm{BMI} \geq 30 \mathrm{~kg} / \mathrm{m}^{2}\right)$} \\
\hline & \multicolumn{2}{|c|}{ Black (n 102) } & \multicolumn{2}{|c|}{ White $(n 71)$} & \multicolumn{2}{|c|}{ Black (n 117) } & \multicolumn{2}{|c|}{ White (n 41) } \\
\hline & Mean & $\mathrm{SD}$ & Mean & SD & Mean & SD & Mean & SD \\
\hline Age (years) & $24^{*} \dagger$ & 6 & $30 * \ddagger$ & 8 & $30+\S$ & 8 & $35 \ddagger \S$ & 8 \\
\hline Height (cm) & $161 \cdot 0^{*}$ & $6 \cdot 0$ & $167^{*}$ & $6 \cdot 0$ & $160.0 \S$ & 6.0 & $166 \S$ & $6 \cdot 0$ \\
\hline Weight (kg) & $57.5 \dagger$ & $6 \cdot 2$ & $61 \cdot 2 \ddagger$ & $6 \cdot 9$ & $92.0 \S$ & $14 \cdot 7$ & $95 \cdot 7 \ddagger$ & $14 \cdot 1$ \\
\hline $\mathrm{BMI}\left(\mathrm{kg} / \mathrm{m}^{2}\right)$ & $22.3 \dagger$ & 1.8 & $22 \cdot 0 \ddagger$ & 1.9 & $36 \cdot 0 \dagger \S$ & $5 \cdot 2$ & $34.5 \ddagger \S$ & 4.0 \\
\hline DXA (BF \%) & $30 \cdot 0^{*} \dagger$ & 4.6 & $27 \cdot 9^{\star} \ddagger$ & $5 \cdot 6$ & 45.3† & $4 \cdot 4$ & $44 \cdot 3 \ddagger$ & $2 \cdot 5$ \\
\hline NIR (BF \%) & $25 \cdot 6^{\star} \dagger$ & 4.0 & $27 \cdot 6^{\star} \ddagger$ & $4 \cdot 7$ & $41 \cdot 8+\S$ & $4 \cdot 0$ & $45 \cdot 1 \neq \S$ & 3.7 \\
\hline
\end{tabular}

DXA, dual-energy X-ray absorptiometry; NIR, near-IR reactance; $\mathrm{BF} \%$, body fat percent.

$P$ values are adjusted for age.

${ }^{*}$ Normal-weight black $v$. normal-weight white $(P<0.05)$.

† Normal-weight black $v$. obese black $(P<0.05)$.

$\ddagger$ Normal-weight white $v$. obese white $(P<0.05)$.

$\S$ Obese black $v$. obese white $(P<0.05)$. 
subjects were older and had a greater body weight, higher BMI and increased adiposity compared to the normal-weight subjects. There were no differences in height between the normal-weight and obese women of the same ethnicity.

The white subjects were older, taller and heavier than black subjects $(P<0 \cdot 05)$. There were no ethnic differences in BMI in normal-weight women $(P=0 \cdot 20)$; however, in obese women, BMI was higher in black compared to white women $(P<0.05)$. In normal-weight women, white women had a lower DXA- and NIR-derived BF\%, than black women $(P<0.01)$. However, in obese women, BF\% measured by DXA was greater in black compared to white women $(P<0 \cdot 01)$, but when measured by NIR, BF \% was greater in white compared to black women $(P<0 \cdot 05)$.

\section{Bivariate relationship between near-IR reactance-and dual-energy $X$-ray absorptiometry-derived measures of body fat percent}

The bivariate relationship between NIR- and DXA-derived measures of $\mathrm{BF} \%$ for each group is shown in Fig. 1. NIRderived $\mathrm{BF} \%$ correlated significantly with DXA-derived measures in both normal-weight and obese black women ( $r$ 0.55, 95\% CI: $0.40,0.67, P<0.001$ and $r 0.59$, $95 \% \mathrm{CI}$ : $0.44,0.68, P<0.001$, respectively) and normal-weight and obese white women $(r 0.69,95 \%$ CI: $0.53,0.79, P<0.001$ and $r 0.56,95 \% \mathrm{CI}: 0.30,0.74, P<0.001$, respectively). In obese subjects, NIR-derived $\mathrm{BF} \%$ did not measure values greater than approximately $45 \%$, while the maximum DXAderived measure was $58 \%$. Using DXA, $18 \%$ of white women and $26 \%$ of black women had $\mathrm{BF} \%>45 \%$, resulting in a large under-prediction of $\mathrm{BF} \%$ by NIR in these women.

\section{Limits of agreement between near-IR reactance- and dual-energy $X$-ray absorptiometry-derived measures of body fat percent}

The Bland-Altman limits of agreement between NIR- and DXA-derived BF \% are shown in Fig. 2(a) and (b) for the black and white subjects, respectively. NIR under-predicted $\mathrm{BF} \%$ compared to DXA in black women (95\% CI for the mean difference: normal-weight, $-5 \cdot 2$ to -3.6 and obese, -4.1 to -2.7$)$ but not in white women $(95 \% \mathrm{CI}$ : normal-weight, -1.3 to 0.7 and obese, -1.8 to 0.2 ). There were no differences in the bias between the normal-weight and obese black $(P=0.54)$ or white women $(P=0.99)$. There was no observable pattern in the variation between $\mathrm{BF} \%$ measured by NIR and DXA up to $45 \%$ body fat, beyond which NIR under-predicted BF \%.

\section{Discussion}

Although single-site NIR is a practical field method for the measurement of body composition, it under-predicted BF \% in normal-weight and obese black South African women compared to DXA. The degree of under-prediction was much greater in subjects with very high levels of adiposity $(>45 \%)$. BF\% measured by NIR appeared to level off at approximately $45 \%$, while DXA measured up to almost $60 \%$ body fat. The degree of under-prediction of $\mathrm{BF} \%$ by single-site NIR could be related to several methodological limitations, including the impact of skin colour ${ }^{(19)}$, body fat distribution patterns ${ }^{(11)}$ and measurement site $^{(20)}$ on NIR.

There is a paucity of data available on the use of NIR in black Africans and other ethnic groups. We show that NIR under-predicts BF\% significantly more in black (approximately $4 \%$ ) compared to white (approximately $<1 \%$ ) women, irrespective of body size. Similarly, Hicks et al. ${ }^{(11)}$ reported that single-site NIR under-estimated adiposity by an average of $4.4 \%$ compared to underwater weighing in 146 American Indian women (age 34.3 (SD 10) years; BMI 25.5 $(\mathrm{SD} 4.3) \mathrm{kg} / \mathrm{m}^{2}$ ). However, these results were not compared to another ethnic group of similar body composition. The impact of skin tone on optical density measures, the basis of the NIR methodology, could possibly explain these findings. In a cohort of white, black, Hispanic and American Indian men ( $n$ 150), skin tone explained a significant amount of the variability in optical density at the biceps site $(16 \%)$, beyond that explained by skinfold thickness alone $(41 \%)$. Moreover, individuals with darker skin colours tended to have a smaller change in optical density, indicative of decreased subcutaneous adiposity compared to those with
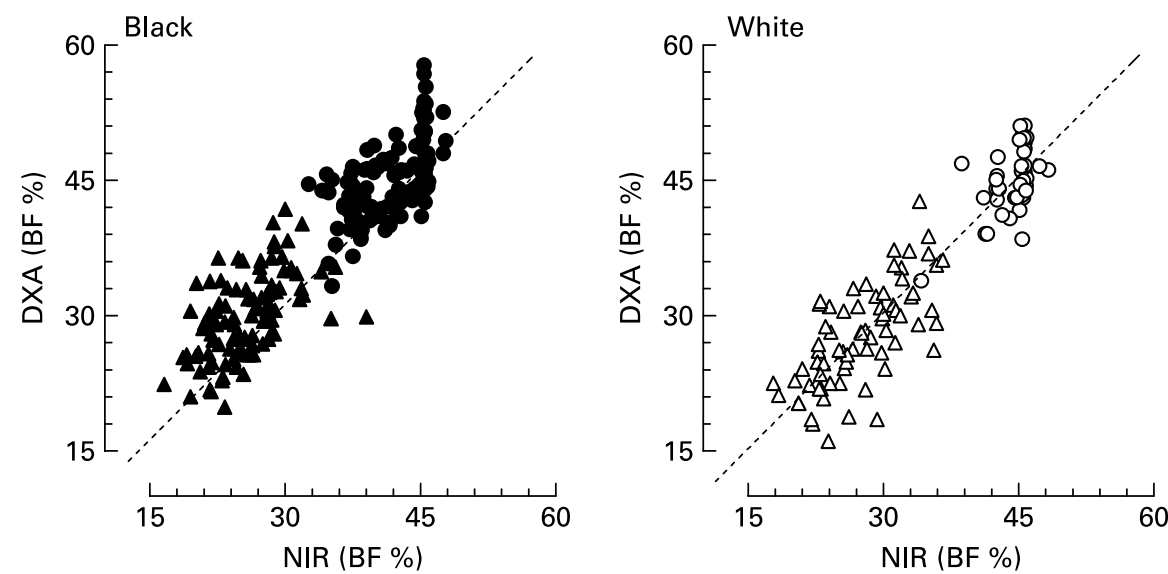

Fig. 1. The correlation between near-IR reactance (NIR)- and dual-energy X-ray absorptiometry (DXA)-derived measures of body fat percent (BF \%) in black and white, normal-weight and obese South African women. $\bigcirc$, $\bullet$, Obese subjects; $\Delta, \boldsymbol{\Delta}$, normal-weight subjects. $\bullet, \boldsymbol{\Delta}$ Black women; $\bigcirc$, $\triangle$, white women; --, line of identity. 

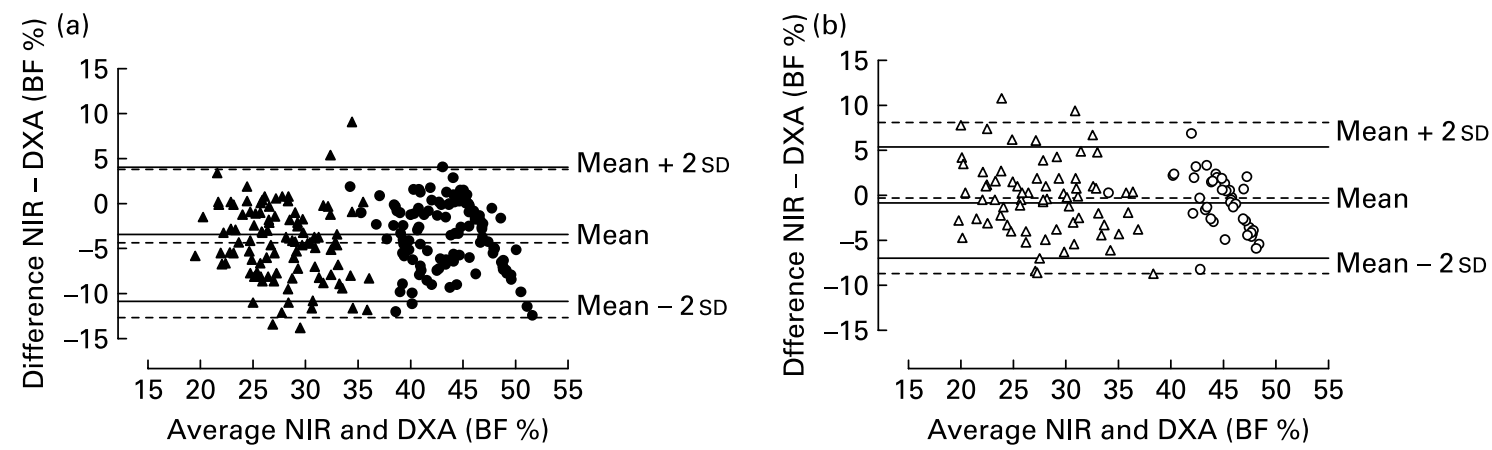

Fig. 2. The Bland-Altman limits of agreement (LOA) between body fat percent (BF\%) measured by near-IR reactance (NIR) and dual-energy X-ray absorptiometry (DXA) in normal-weight and obese, black (a) and white (b) South African women. $\bigcirc, \bullet$, Obese subjects; $\triangle, \mathbf{\Lambda}$, normal-weight subjects. $\bullet, \mathbf{\Delta}$ Black women; $O, \triangle$, white women; --, LOA for the normal-weight women; - , obese women.

lighter skin colours ${ }^{(19)}$. Therefore, darker skin colour could impact on the relationship between $\mathrm{BF} \%$ and optical density and confound the use of NIR in this population. Melanin has been shown to affect absorption and/or reflectance of light at low wavelengths ${ }^{(21)}$, however, little is known about this effect at higher wavelengths $(>700 \mathrm{~nm})$ that are used for the measurement of $\mathrm{BF} \%$ by NIR. If melanin is found to have an effect at higher wavelengths, the inclusion of a measure of light absorption/reflectance in the NIR algorithm should be considered.

Alternatively, these differences may also be explained, in part, by ethnic differences in body fat distribution, as singlesite NIR is measured at one focused point on the bicep, and therefore may not be a sensitive measure of whole $\mathrm{BF} \%$. Ethnic differences in body fat distribution have been reported $^{(7)}$, with black South Africans and African Americans having less visceral fat and more peripheral subcutaneous fat than their white counterparts ${ }^{(13-15)}$. Hicks et al. ${ }^{(11)}$ proposed that NIR under-predicted $\mathrm{BF} \%$ in American Indian women due to differences in body fat distribution compared to white populations, on which NIR (Futrex-5000) equations were determined. Moreover, by incorporating hip circumference, biceps and chest optical density, physical fitness, age and height into a regression equation, $79 \%$ of the variance in $\mathrm{BF} \%$ in the American Indian women could be accounted for compared to only $36 \%$ using single-site NIR (biceps optical density) and $62 \%$ using multi-site NIR (abdominal, subscapular, thigh, chest and biceps optical density $)^{(11)}$. Since black women have decreased centralisation of body fat and increased peripheral fat mass compared to their white counterparts $^{(7)}$, perhaps use of the biceps as the NIR measurement site, which was shown to be the best predictor of total BF\% $\left(r\right.$ 0.85) in middle-aged white men ${ }^{(20)}$, is not appropriate in black women.

The inability of NIR to accurately measure BF \% is not unexpected when one considers the lessons learned from other body composition methods that extrapolate total adiposity from a measurement of one body segment. For example, the skinfold thickness method operates on the assumption that subcutaneous adipose tissue is representative of total body fat and that fat-free mass density is uniform in all populations. Further, the NIR beam only penetrates to a depth of approximately $1 \mathrm{~cm}$. Therefore, differences in total adiposity and body fat distribution may affect the validity of the measurement. This has resulted in the development of over 100 population-specific equations with correlation coefficients for women ranging from $r$ 0.72-0.84, compared to criterion methods ${ }^{(22)}$. Just as there are population-specific equations for fat predicted from skinfold measurements, it is likely that population-specific equations for single-site NIR should be derived and validated to account for ethnic variations in body fat distribution patterns. This is highlighted by the fact that BMI, a gross estimate of body fatness was a better correlate of DXA BF\% than NIR in obese black women $(r 0.75, P<0.001)$. In contrast, the correlation between NIR and DXA BF \% was stronger than BMI in normal-weight black $(r 0.46, P<0.001)$ and white women $(r 0.63, P<0.001)$, and obese white women $(r 0.53, P<0.001)$.

When comparing normal-weight and obese individuals within each ethnic group, we found a similar magnitude of difference in BF \% between NIR- and DXA-derived measures, with NIR under-estimating BF\% equally in normal-weight and obese women. Other studies have shown that NIR consistently under-predicts $\mathrm{BF} \%$ in obese individuals ${ }^{(23,24)}$, with the degree of under-estimation increasing with increasing levels of adiposity ${ }^{(25)}$. This is confirmed in the present study; in particular as NIR appeared to level off at approximately $45 \%$, while DXA measured up to almost $60 \%$ body fat. NIR may therefore be inappropriate for use in very obese populations. The findings of studies investigating the validity of NIR in non-obese individuals are not as consistent as in obese individuals. A study reported that NIR accurately estimated $\mathrm{BF} \%$ in non-obese and lean individuals compared to underwater weighing ${ }^{(24)}$, while a more recent study reported that NIR, when compared to underwater weighing, overestimated $\mathrm{BF} \%$ in lean individuals ${ }^{(10)}$. In contrast, we found that NIR under-predicted BF \% in the black, but not white normal-weight subjects.

To our knowledge, this is the first study investigating the use of NIR in black and white South African women, and is the largest study to date investigating NIR as a measure of body composition in black African individuals. We do, however, acknowledge the limitations of a single-site NIR measurement. However, we chose single-site NIR in order to test the accuracy of an economical (time and money) field measure of $\mathrm{BF} \%$ that would be applicable to a large scale study. Furthermore, Hortobágyi et al. ${ }^{(26)}$ have shown that NIR measured at multiple sites does not improve the measurement of $\mathrm{BF} \%$. Most of the previous studies have used underwater weighing as the criterion measure when 
validating NIR. However, as DXA is widely accepted as a state-of-the-art tool to measure body composition, it was chosen as the criterion method for the present study.

In conclusion, the results of the present study suggest that single-site NIR should be used with caution in women of African descent and individuals with high levels of adiposity. Furthermore, investigation of ethnic-specific field techniques for quantifying body composition in individuals with varying levels of adiposity, as well as longitudinal intervention studies examining changes in body fat are required for the purposes of both health risk assessment and research.

\section{Acknowledgements}

We thank the research volunteers for their participation in the present study and Nandipha Sinyanya for her excellent field work. Linda Bewerunge is thanked for performing the DXA scans. The present study was funded by the South African Medical Research Council, the International Atomic Energy Agency, the National Research Foundation of South Africa and the University of Cape Town. The authors declare no conflict of interest. Author contributions. C. L. J. and L. K. M. did data collection, analysis and manuscript preparation. M. I. L. did study conception, data analysis and manuscript preparation. E. V. L. did study conception, funding, data analysis and manuscript preparation. M. C. did manuscript preparation. J. H. G. did funding, data analysis and manuscript preparation.

\section{References}

1. WHO (2000) Obesity: preventing and managing the global epidemic. Obesity. Technical Report Series no. 894. Geneva: WHO.

2. Puoane T, Steyn K, Bradshaw D, et al. (2002) Obesity in South Africa: The South African demographic and health survey. Obes Res 10, 1038-1048.

3. Bray GA (2004) Medical consequences of obesity. J Clin Endocrinol Metab 89, 2583-2589.

4. Chang CJ, Wu CH, Chang CS, et al. (2003) Low body mass index but high percent body fat in Taiwanese subjects: implications of obesity cutoffs. Int J Obes Relat Metab Disord 27, 253-259.

5. Ito H, Nakasuga K, Ohshima A, et al. (2003) Detection of cardiovascular risk factors by indices of obesity obtained from anthropometry and dual-energy X-ray absorptiometry in Japanese individuals. Int J Obes Relat Metab Disord 27, 232-237.

6. Fernandez JR, Heo M, Heymsfield SB, et al. (2003) Is percentage body fat differentially related to body mass index in Hispanic Americans, African Americans, and European Americans? Am J Clin Nutr 77, 71-75.

7. Rush EC, Goedecke JH, Jennings C, et al. (2007) BMI, fat and muscle differences in urban women of five ethnicities from two countries. Int J Obes (Lond) 31, 1232-1239.

8. Kiebzak GM, Leamy LJ, Pierson LM, et al. (2000) Measurement precision of body composition variables using the lunar DPX-L densitometer. J Clin Densitom 3, 35-41.
9. Ward A, Pollock ML, Jackson AS, et al. (1978) A comparison of body fat determined by underwater weighing and volume displacement. Am J Physiol 234, E94-E96.

10. Daniel JA, Sizer PS Jr \& Latman NS (2005) Evaluation of body composition methods for accuracy. Biomed Instrum Technol 39, 397-405.

11. Hicks VL, Stolarczyk LM, Heyward VH, et al. (2000) Validation of near-infrared interactance and skinfold methods for estimating body composition of American Indian women. Med Sci Sports Exerc 32, 531-539.

12. Hortobagyi T, Israel RG, Houmard JA, et al. (1992) Comparison of four methods to assess body composition in black and white athletes. Int J Sport Nutr 2, 60-74.

13. Goedecke JH, Levitt NS, Lambert EV, et al. (2009) Differential effects of abdominal adipose tissue distribution on insulin sensitivity in black and white South African women. Obesity (Silver Spring) 17, 1506-1512.

14. Lovejoy JC, de la Bretonne JA, Klemperer M, et al. (1996) Abdominal fat distribution and metabolic risk factors: effects of race. Metabolism 45, 1119-1124.

15. Lovejoy JC, Smith SR \& Rood JC (2001) Comparison of regional fat distribution and health risk factors in middle-aged white and African American women: The Healthy Transitions Study. Obes Res 9, 16.

16. Panotopoulos G, Ruiz JC, Guy-Grand B, et al. (2001) Dual $\mathrm{X}$-ray absorptiometry, bioelectrical impedance, and near infrared interactance in obese women. Med Sci Sports Exerc 33, 665-670.

17. Micklesfield LK, Reid S, Bewerunge L, et al. (2007) A proposed method to measure body composition in obese individuals using dual-energy X-ray absorptiometry. Int J Body Compos Res 5, $147-151$.

18. Bland JM \& Altman DG (1986) Statistical methods for assessing agreement between two methods of clinical measurement. Lancet 8, 307-311.

19. Wilson WL \& Heyward VH (1993) Validation of the near-infrared interactance method for Native Indian, black, Hispanic and white men 19 to 57 years. Basic Life Sci 60, 389-392.

20. Durrett M \& Thorland W (1990) Reliability and validity of multisite infrared interactance measures of body density. Med Sci Sports Exerc 25, Abstract 337.

21. Anderson RR \& Parrish JA (1981) The optics of human skin. J Invest Dermatol 77, 13-19.

22. Brodie D, Moscrip V \& Hutcheon R (1998) Body composition measurement: a review of hydrodensitometry, anthropometry, and impedance methods. Nutrition 14, 296-310.

23. McLean KP \& Skinner JS (1992) Validity of Futrex-5000 for body composition determination. Med Sci Sports Exerc 24, $253-258$

24. Heyward VH, Cook KL, Hicks VL, et al. (1992) Predictive accuracy of three field methods for estimating relative body fatness of nonobese and obese women. Int J Sport Nutr 2, 75-86.

25. Elia M, Parkinson SA \& Diaz E (1990) Evaluation of near infrared interactance as a method for predicting body composition. Eur J Clin Nutr 44, 113-121.

26. Hortobágyi T, Israel RG, Houmard JA, et al. (1992) Comparison of body composition assessment by hydrodensitometry, skinfolds, and multiple site near-infrared spectrophotometry. Eur J Clin Nutr 46, 205-211. 